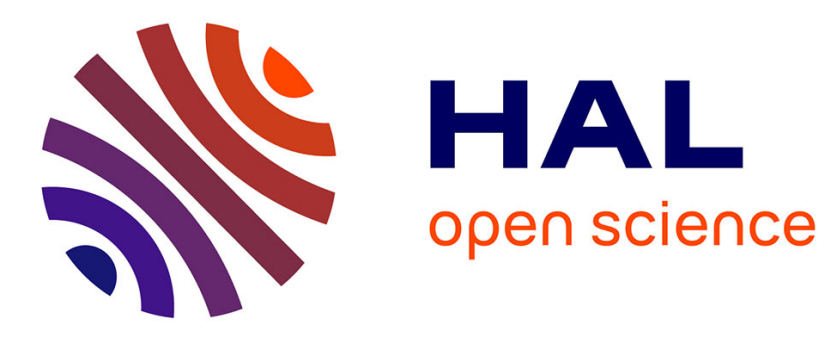

\title{
Estimating Scalable Common-Denominator Laplace-Domain MIMO Models in an Errors-in-Variables Framework
}

Gerd Vandersteen, Ludwig De Locht, Snezana Jenei, Yves Rolain, Rik Pintelon

\section{To cite this version:}

Gerd Vandersteen, Ludwig De Locht, Snezana Jenei, Yves Rolain, Rik Pintelon. Estimating Scalable Common-Denominator Laplace-Domain MIMO Models in an Errors-in-Variables Framework. DATE'05, Mar 2005, Munich, Germany. pp.1076-1081. hal-00181682

\section{HAL Id: hal-00181682 \\ https://hal.science/hal-00181682}

Submitted on 24 Oct 2007

HAL is a multi-disciplinary open access archive for the deposit and dissemination of scientific research documents, whether they are published or not. The documents may come from teaching and research institutions in France or abroad, or from public or private research centers.
L'archive ouverte pluridisciplinaire HAL, est destinée au dépôt et à la diffusion de documents scientifiques de niveau recherche, publiés ou non, émanant des établissements d'enseignement et de recherche français ou étrangers, des laboratoires publics ou privés. 


\section{Estimating scalable common-denominator Laplace-domain MIMO models in an errors-in-variables framework}

\author{
Gerd Vandersteen, Ludwig De Locht, Snezana Jenei \\ IMEC vzw. \\ Kapeldreef 75 \\ B-3001 Leuven, Belgium
}

\author{
Yves Rolain, Rik Pintelon \\ Vrije Universiteit Brussel \\ Dept. ELEC \\ Pleinlaan 2 \\ B-1050 Brussels, Belgium
}

\begin{abstract}
Design of electrical systems demands simulations using models evaluated in different design parameters choices. To enable the simulation of linear systems, one often requires their modeling as ordinary differential equations given tabular data obtained from device simulations or measurements. Existing techniques need to do this for every choice of design parameters since the model representations dont scale smoothly with the external parameter.

The paper describes a frequency-domain identification algorithm to extract the poles and zeros of linear MIMO systems. Furthermore, it expresses the poles and zeros as trajectories that are functions of the design parameter(s). The paper describes the used framework, solves the startingvalue problem, presents a solution for high-order systems and provides a model-order selection strategy. The properties of the algorithm are illustrated on microwave measurements of inductors, a variable gain amplifier and a highorder SAW-filter. As shown by these examples, the proposed identification algorithm is very well suited to derive scalable, physically relevant models out of tabular frequencyresponse data.
\end{abstract}

\section{Introduction}

The design and simulation of high-performance electronic systems requires accurate and efficient models. The model requirements depend on the system type and on how the model is used in the design flow. The use of the model in an actual design requires that the model is parameterisable as function of external parameters. This makes it possible to predict the model of a system which was not simu-

* This research was supported by the Fund for Scientific Research, Flanders, the Flemish government (GOA-ILINOS), and the Belgian program on Inter-university Poles of attraction (IUAP-5/22). lated/measured yet. A practical example is the prediction of an inductor model out of measurements of related - though differently sized inductors. This will be highlighted in the examples given later.

The modeling capabilities differ depending on the initial available model or data. Model reduction techniques determine a simplified model starting from known model equations [4, 9]. Identification techniques start from the system response only, without the knowledge of the systems equations $[2,10]$. An example of the latter is the identification of linear systems using observed inputs and outputs or using the transfer matrices obtained through simulations or measurements.

Scalable models need to describe physically relevant model properties. Poles and zeros of a linear system satisfy this constraint. This is why the pole/zero trajectories will be parameterized instead of the coefficients of a rational form. The use of an invariant guarantees that the dependency on the external parameter(s) is physically relevant.

Passivity will not be imposed in this work for two reasons. First, making a non-passive model passive implies that pole/zero locations are artificially modified which compromises the physical relevance of the scalable model. Second, it is possible to impose stability in a post-processing stage: A passive model can be obtained from a stable system using convex optimization [3].

We propose a robust and efficient identification algorithm to extract a scalable model for Multiple-Input Multiple-Output (MIMO) linear systems starting from tabular frequency-dependent data. The parameterized MIMO linear model determines the pole/zero trajectories starting from a common-denominator transfer-matrix model. Such models can be used in SPICE-like transient simulators such as Spectre.

MIMO linear systems, described by ordinary differential equations, can be represented using a common-denominator transfer-matrix representation. This means that the exact inputs $U_{j}^{0}(s)$ with $j=1 \ldots n_{U}$ and the exact outputs $Y_{i}^{0}(s)$ 
with $i=1 \ldots n_{Y}$ are related to each other using

$$
Y_{i}^{0}(s)=\sum_{j=1}^{n_{U}} \frac{N_{i j}(s)}{d(s)} U_{j}^{0}(s)
$$

where $s$ represent the Laplace variable, $d(s)$ the denominator polynomial and $N_{i j}(s)$ the numerator polynomials. All polynomials have real-valued coefficients. The poles of the system are presented by the zeros of the common denominator $d(s)$. If some poles are not observable in certain transfer functions, then the influences of these poles are compensated by zeros. This does not lead to numerical conditioning problems if the appropriate orthogonal polynomials are used.

An errors-in-variables framework is used to derive the estimators. This implies that both the inputs and outputs are perturbed with zero-mean additive noise $\Delta U_{i}(s)$ and $\Delta Y_{j}(s)$

$$
U_{i}=U_{i}^{0}+\Delta U_{i} ; Y_{j}=Y_{j}^{0}+\Delta Y_{j}
$$

with the noise covariance equal to

$$
\begin{aligned}
\sigma_{U_{i} U_{j}}^{2}(s) & =\mathrm{E}\left[\overline{\Delta U_{i}(s)} \Delta U_{j}(s)\right] \\
\sigma_{U_{i} Y_{j}}^{2} & (s)=\mathrm{E}\left[\overline{\Delta U_{i}(s)} \Delta Y_{j}(s)\right] . \\
\sigma_{Y_{i} Y_{j}}^{2}(s) & =\mathrm{E}\left[\overline{\Delta Y_{i}(s)} \Delta Y_{j}(s)\right]
\end{aligned}
$$

Some of these (co-)variances can be equal to zero depending on the data source and the wanted approximation. Therefore, all identification steps are made robust to such degenerated cases.

The two contributions of the paper are: setting up a robust MIMO-identification algorithm and demonstrating the modeling the pole/zero trajectories as function of external parameters.

The first contribution of this paper is to set up an efficient and robust MIMO identification algorithm in the errors-invariables framework. This requires the extension of several Single-Input Single-Output (SISO) solutions towards MIMO and demands a careful selection of the successive identification steps to obtain a robust identification algorithm. Section 2 describes the state-of-the-art, the required MIMO extension and the motivated selection of the identification steps to obtain a robust identification algorithm.

The second contribution of the paper is to demonstrate the modeling of the pole/zero trajectories as function of external parameters. Section 3 therefore gives an answer to the question: What is the most appropriate parameterization for modeling the pole/zero trajectories ?

The identification algorithm is illustrated in Section 4 using the following three examples: extracting a scalable inductor model starting from measurements, modeling a Variable Gain Amplifier (VGA) with respect to its gain setting and modeling a SAW-filter. All these results demonstrate the ability of estimating physically relevant pole/zero trajectories for (potentially high-order) linear systems, which is concluded in Section 5.

\section{Frequency domain identification tech- niques}

A high-performance identification technique for identifying high-order MIMO linear models must satisfy that

1. the class of models and the identification framework are according to the problem;

2. good starting values are provided for the final nonlinear minimization;

3. high-order systems can be handled without numerical conditioning problems;

4. a model selection strategy is available.

A robust, high-performance identification algorithm is presented after describing the state-of-the-art and the necessary extensions.

\subsection{Identification framework}

The framework chosen to identify the MIMO linear systems is a maximum-likelihood (ML) estimator under the assumption that

- the linear system is represented using a commondenominator transfer matrix (1);

- both the inputs and the outputs are perturbed with additive noise (2);

- the uncertainties on inputs and outputs can be correlated and frequency dependent (3).

The maximum-likelihood estimate can be derived under the assumption that the noise is independent over the $K$ frequencies and normally distributed with given covariance matrix [10]. The leads to the minimization of the ML cost,

$$
L_{\mathrm{ML}}(p)=\sum_{k=1}^{K} e_{k}^{H} C_{e_{k}}^{-1} e_{k}=\sum_{k=1}^{K} r_{k}^{H} r_{k},
$$

with respect to the real-valued parameter vector

$$
p^{T}=\left[\begin{array}{llll}
p_{N_{11}}^{T} & \cdots & p_{N_{n_{Y} n_{U}}}^{T} & p_{d}^{T}
\end{array}\right] .
$$

Vector $e_{k}=e\left(p, \omega_{k}\right)$ represent the error vector which equals to

$$
\sum_{j=1}^{n_{U}} N_{i j}\left(p_{N_{i j}}, \omega_{k}\right) U_{j}\left(\omega_{k}\right)-d\left(p_{d}, \omega_{k}\right) Y_{i}\left(\omega_{k}\right)
$$

with $N_{i j}\left(p_{N_{i j}}, \omega_{k}\right)=P_{N_{i j}}(j \omega) p_{N_{i j}}$ and $d\left(p_{d}, \omega_{k}\right)=$ $P_{d}(j \omega) p_{d}$ where $P$. represents the polynomial basis used. 
Matrix $C_{e_{k}}=C_{e\left(p, \omega_{k}\right)}$ is the covariance matrix associated with $e\left(p, \omega_{k}\right)$.

The ML cost can be minimized using standard GaussNewton-like minimization algorithms $[5,8]$ with the residual vector $r_{k}$ given by

$$
r_{k}=C_{e_{k}}^{-1 / 2} e_{k}
$$

Gauss-Newton-like optimization methods demand the computation of the first derivative of the complex-valued $r_{k}$ with respect to the real-valued $p$. This Jacobian matrix $J$ is formed by stacking the matrices

$$
J_{k}=\left[\begin{array}{lll}
\frac{\partial r_{k}}{\partial p_{1}} & \cdots & \frac{\partial r_{k}}{\partial p_{n_{p}}}
\end{array}\right]
$$

on top of each other. Generally, the analytic computation of the Jacobian is extremely difficult since it demands the derivative of $C_{e_{k}}^{-1 / 2}$. Analytical expressions are only convenient if $C_{e_{k}}$ is reduced to a scalar or to a diagonal form. However, it is possible to use a pseudo-Jacobian which approximates the Jacobian such that their Gauss-Newton minimizers are identical [8]. This pseudo-Jacobian is compute as

$$
J_{+k}=\left[\begin{array}{lll}
j_{+k, 1} & \cdots & j_{+k, p}
\end{array}\right],
$$

with

$$
j_{+k, i}=C_{e_{k}}^{-1 / 2} \frac{\partial e_{k}}{\partial p_{i}}-\frac{1}{2} C_{e_{k}}^{-1 / 2} \frac{\partial C_{e_{k}}}{\partial p_{i}} C_{e_{k}}^{-1} e_{k} .
$$

\subsection{Solving the starting value problem}

This section describes two algorithms - a weighted linear least-squares (WLLS) and a weighted generalized totalleast-squares (WGTLS) - that can be used to generate starting values for $p$. The starting values depend on the polynomial bases, $P .\left(j \omega_{k}\right)$, and on the weighting, $W$, used. The polynomial bases used are described in Section 2.3.

A first starting value solution is to linearize (7) by taking $C_{e_{k}}^{-1 / 2}$ constant which results in a WLLS estimator. Since $C_{e_{k}}^{-1 / 2}$ depends on the (unknown) parameters $p$, it is necessary to determine the initial weighting empirically. This empirical weighting can only use the measured transfer matrices and the asymptotic behavior of the polynomials, given the orders of the polynomials. Such weighting has been proposed for the SISO case [12]. In our MIMO case, we use the empirical weighting of [12] for each transfer function separately and sum the contributions together for each output.

An alternative to the WLLS is to use a WGTLS estimator $[10,14]$. Observing that the error vector (6) is linear in the parameters makes it possible to write $e=A p$ with

$$
A=\left[\begin{array}{ccccc}
P_{N_{11}} U_{1} & 0 & \cdots & 0 & -P_{d} Y_{1} \\
0 & P_{N_{12}} U_{1} & \cdots & 0 & -P_{d} Y_{2} \\
\vdots & \vdots & \ddots & \vdots & \vdots \\
0 & 0 & \cdots & P_{N_{n_{U} n_{Y}}} U_{n_{U}} & -P_{d} Y_{n_{Y}}
\end{array}\right]
$$

The solution of the WGTLS is given by the singular value decomposition (SVD) of the matrix $W A C^{-1}$ were $W$ and $C$ are weighting matrices mandatory to obtain consistent and nearly ML-efficient estimates [7, 10]. The matrix $W$ weights the importance of each equation and therefore determines the efficiency of the estimator. Optimally, $W$ equals $C_{e}^{-1 / 2}$. The weighting matrix $C$ makes the estimates consistent if $C^{H} C=E\left[(W A)^{H}(W A)\right]$ [14]. Matrix $C$ becomes singular when some of the inputs or outputs are known exactly. We therefore advise to compute the WGTLS solution using a generalized SVD (GSVD) [14]. This avoids the inversion of the - possibly singular - matrix $C$.

The WLLS and the WGTLS can also be used iteratively by plugging previous estimates of $p$ into the weighting. Such WGTLS iterative estimators - known as the Bootstrapped Total Least-Squares (BTLS) estimator - generates strongly consistent estimates at each iteration [14].

Restarting the estimation with an improved weighting is required in three situations

1. If no nonlinear optimizer is available, then the parameters can be estimated by the iterative use of the WLLS or the BTLS.

2. If the ML nonlinear optimizer gets stuck into a local minima, then we advice to use the BTLS estimator to generate an alternative starting point. The BTLS estimator is more appropriate than the WLLS since the latter is a linearized version of the ML cost. Hence, the probability that the WLLS estimate gets stuck in the neighborhood of the nonlinear optimizer is significantly higher.

3 . If the conditioning severely decreases during the optimization of the ML-cost, then a re-orthogonalization is required by restarting with the WLLS or the BTLS estimator using a weighting evaluated in the previous ML-estimates.

\subsection{Forsythe orthogonal polynomial enable high- order models}

Several strategies are proposed in the literature to improve the numerical conditioning of weighted $[1,6,13]$ and unweighted least-squares problems [2]. Optimal conditioning for polynomial models require Forsythe orthogonal polynomials which make $J^{H} J$ equals the identity for the WLLS and the WGTLS [6]. They are constructed using a 3-term recurrence algorithms for both real-valued polynomials evaluated over the real axis [6] and for complexvalued polynomials evaluated over the imaginary axis [13].

Optimal conditioning for rational models can be obtained using vector orthogonal polynomials [1]. These polynomials mix all basis functions of the denominator and all 
numerators. This makes the use of vector orthogonal polynomials significantly more complex and computationally slower.

A practical alternative for the complete orthogonalization uses the Forsythe orthogonal polynomials to represent the individual polynomials. Rational approximations using Forsythe orthogonal polynomials lead to the bestconditioned representation and are demonstrated up to order 120 [13].

\subsection{Model selection problem for MIMO}

A model selection algorithm is required to determine the orders of the numerators $N_{i j}(s)$ and the denominator $d(s)$. The model selection algorithm is based on the SISO model selection algorithm of [11]. This algorithm is a two-step order selection method with a coarse and a fine selection.

The coarse selection starts with an over-estimate of the order. It uses the singular values of a whitened Jacobian matrix to determine a (slight) over-estimate of the order [11]. Whitening the Jacobian is necessary to set the threshold for the selection procedure.

The fine selection is performed through elimination of poles/zeros without significant contribution to the model in the frequency band of interest. The fine selection for SISO models [11] is adapted when considering the distance between poles and zeros. A pole/zero pair cancels in the SISO case if their distance equals zero. This is generalized to MIMO by considering the distance between a pole and the zeros of each individual transfer function. A pole/zeros cancels if the distances of a pole to a zero in all numerators $N_{i j}(s)$ equal zero.

\subsection{The proposed identification algorithm}

An identification Algorithm 1 is based on the results of the previous sections. Its main properties are:

- each iteration uses a BTLS estimator as starting value generator. BTLS is preferred over WLLS since it is observed that the algorithm becomes less sensitive to local minima.

- the initial weighting $W$ for the BTLS estimator uses the empirical algorithm based on the measured transfer matrix and the orders of the polynomials.

- each iteration restarts with a new weighting which converges to the optimal ML-weighting.

- each iteration performs a re-orthogonalization to obtain a good conditioning of the final ML-estimate. Intermediate ML-estimates can suffer from bad conditioning. This occurs when the starting values differ significantly from the ML-estimates.

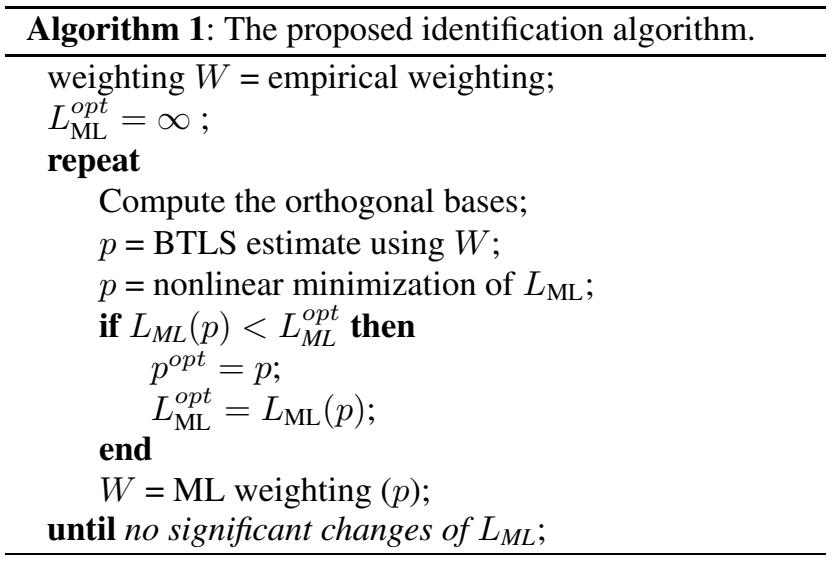

\section{Modeling the poles / zero trajectory}

Pole/zero representations of a linear system are independent of the polynomial bases used. The pole/zero trajectories are therefore a good candidate to model the dependency on external parameter(s).

The modeling of the pole/zero trajectories requires the grouping of the poles and zeros such that they form a smooth trajectory as a function of the external parameter. The results obtained in Section 4 use a nearest neighbor algorithm to determine the pole/zero trajectories.

Another issue is the modeling of the pole/zero trajectories themselves: when two real poles move to each other and become a complex pole-pair, then the derivatives of the trajectory are discontinuous. This problem can be solved by either splitting the trajectory into pieces or by combining pole pairs into second-order sections. The latter strategy used in the below examples - writes the different polynomials as the product of first and second order polynomials.

\section{Examples}

The most important aspects of the proposed algorithm are illustrated on three examples.

A. The extraction of a scalable model from microwave measurements is required when introducing state-of-the-art inductor technology into a spice-like simulator.

The scalable inductor model was extracted for off-chip inductors made in MCM technology. The 1.5-turn inductors had identical inner diameters $(100 \mu \mathrm{m})$ and conductor spacing $(20 \mu \mathrm{m})$. The conductor width $w$ was changed from $50 \mu \mathrm{m}, 77 \mu \mathrm{m}, 100 \mu \mathrm{m}$ till $150 \mu \mathrm{m}$, resulting in nominal inductor values of $1.62,1.74,1.80,2.04 \mathrm{nH}$. All inductors were measured from $45 \mathrm{MHz}$ till $50 \mathrm{GHz}$.

$5^{\text {th }}$ order models were extracted out of the measurements from DC up to two times the frequency of the first minimum in $S_{21}$. This minimum changes from $34 \mathrm{GHz}$ down to respectively $25,20.8$ and $14.8 \mathrm{GHz}$ for the $50 \mu \mathrm{m}, 77 \mu \mathrm{m}$, 


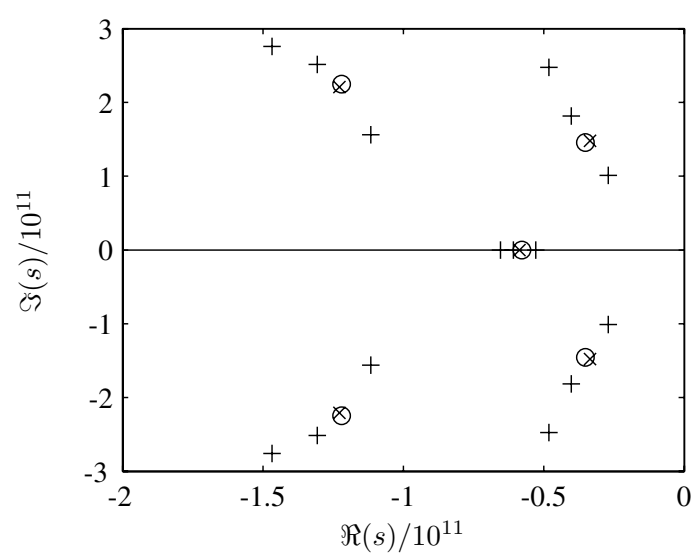

Figure 1. The pole trajectories of a $5^{\text {th }}$ order inductor model. A cubic interpolation is used to predict $(0)$ starting from the estimates $(+)$. Ideally, the interpolations $(\circ)$ coincide with the estimates of the $100 \mu \mathrm{m}$ inductor $(\times)$.
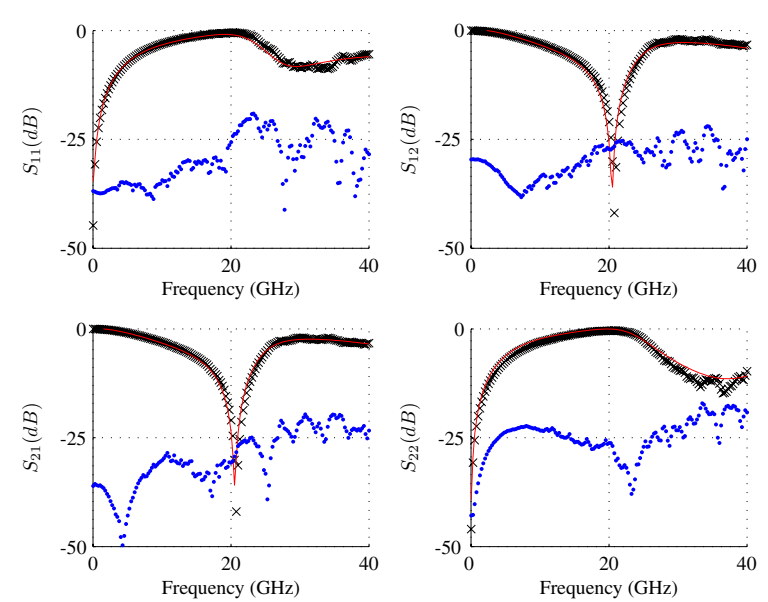

Figure 2. Comparison between the measured transfer function of the $100 \mu \mathrm{m}$ inductor $(\times)$ and the prediction using the scalable inductor model (-). The complex difference (.) shows the excellent agreement between the measurements and the prediction.
$100 \mu \mathrm{m}$ and $150 \mu \mathrm{m}$ inductors. The wide frequency range used implies that the model is valid far beyond the region were it operates as an inductor.

The validation of the scalable model was done as follows:

1. $5^{\text {th }}$ order models were extracted for all inductors and the pole/zero trajectories were determined. The measurement allowed to obtain modeling errors of below $-30 \mathrm{~dB}$ for all extracted models. The trajectories of the poles are illustrated in Figure 1. The trajectories for the zeros are similar but are not shown due to the lack of space.

2. The poles and zeros for the $50 \mu \mathrm{m}, 77 \mu \mathrm{m}$ and $150 \mu \mathrm{m}$ inductors were used as reference to predict the poles and zeros from the $100 \mu \mathrm{m}$ inductor. Figure 1 shows the good correspondence of the predicted poles (using a cubic interpolation) and the estimated poles (obtained through the identification algorithm on the measured $100 \mu \mathrm{m}$ inductor).

3. The difference between the predicted and the measured $\mathrm{S}$-parameters was determined, showing a prediction error of about $-25 \mathrm{~dB}$ as illustrated in Figure 2.

The results in Figure 2 clearly demonstrate that it is possible to extract an accurate scalable model for microwave inductors using the proposed technique.

B. VGA modeling from network analyzer measurements for variable gain settings shows the ability of predicting the pole/zero trajectories of complex systems. The measured device is a three stage $5 \mathrm{GHz}$ BiCMOS VGA mounted on a PCB and connected through $3 \mathrm{~cm}$ long transmission lines. The complete system is modeled from $500 \mathrm{MHz}$ to $14 \mathrm{GHz}$ with a $30^{t h}$ order model, resulting in a relative accuracy of about $-30 \mathrm{~dB}$. The VGA is measured in 9 gain settings (from 5 to $25 \mathrm{~dB}$ ). The pole trajectories are shown in Figure 3 . Half of the poles were used as reference while the remaining poles are used to determine the interpolation error of a cubic interpolation.

C. SAW-filter modeling of $30^{\text {th }}$ order shows the ability of extracting high-order models. The model order selection scheme started at model order 60 without numerical problems. The measured and modeled transfer functions are given in Figure 4, together with the complex modeling errors.

\section{Conclusions}

This paper describes a frequency-domain identification technique to extract the pole/zero trajectories for systems which depend on an external parameter. The identification technique estimates a common-denominator MIMO rational model in an errors-in-variables framework. Solutions are presented to estimate high-order models, to deal with 


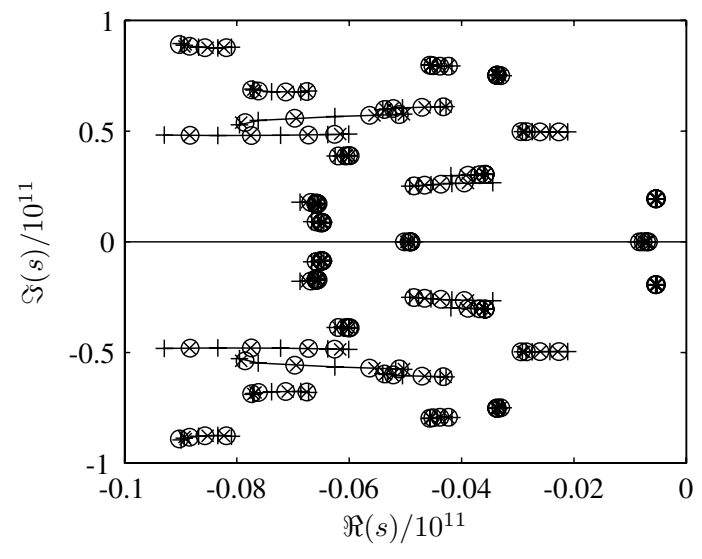

Figure 3. The pole trajectories of a $30^{t h}$ order model for a three-stage VGA with transmission lines included. A cubic interpolation is used to predict $(0)$ starting from the estimates $(+)$. Ideally, the interpolations $(\circ)$ coincide with the estimates $(\times)$.

the starting value problem, to reduce the problem of local minima and to handle the model selection problem.

The proposed algorithm shows excellent results for extracting a scalable model for a microwave inductor and a variable gain amplifier. High-order models can also be handled as shown through the modeling of the SAW-filter. Stable models were extracted in all cases and the pole/zero trajectories were found to be smooth functions of the external parameter.

We therefore recommend the proposed identification algorithm to extract the physically relevant pole/zero information for building scalable models out of tabular simulation or measurement results.

\section{References}

[1] A. Bultheel, M. Van Barel, Y. Rolain, and J. Schoukens. Robust rational approximation for identifi cation. In Proceedings of the 40th IEEE Conference on Decision and Control, volume 5, pages 4770 - 4775, Dec. 2001.

[2] C. Coelho, J. Phillips, and L. Silveira. Robust rational function approximation algorithm for model generation. Proceedings of the 36th Design Automation Conference, June 1999.

[3] C. Coelho, J. Phillips, and L. Silveira. A convex programming approach for generating guaranteed passive approximations to tabulated frequency-data. IEEE Trans. ComputerAided Design, 23(2):293-301, Febr. 2004.

[4] P. Feldmann and R. Freund. Effi cient linear circuit analysis by Pade approximation via the Lanczos process. IEEE Trans. Computer-Aided Design, 14(5):639-649, May 1995.
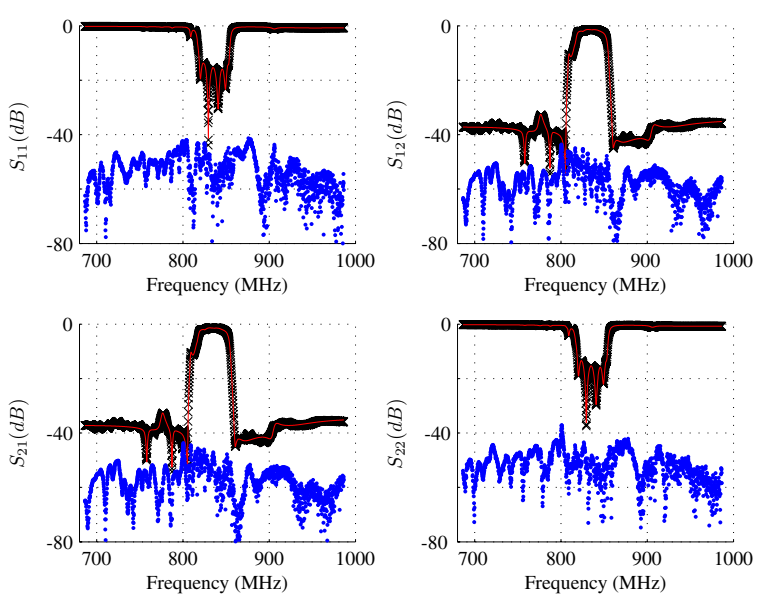

Figure 4. Comparison between the measured transfer function of the SAW-filter $(\times)$ and the $30^{t h}$ order model (-). The complex difference (.) shows the excellent agreement between the data and the model.

[5] R. Fletcher. Practical Methods of Optimization. John Wiley and Sons, New York, 2000.

[6] G. Forsythe. Generation and use of orthogonal polynomials for data fi tting with a digital computer. J. Soc. Indust. Appl. Math., 5:74-88, 1957.

[7] G. Golub and C. Van Loan. Matrix Computations Third Edition. The Johns Hopkins University Press, Baltimore, third edition, 1996.

[8] P. Guillaume and R. Pintelon. A Gauss-Newton-like optimization algorithm for "weighted" nonlinear least-squares problems. IEEE Trans. Sign. Proces., 44(9):2222-2228, Sept. 1196.

[9] A. Odabasioglu, M. Celik, and L. Pileggi. PRIMA: passive reduced-order interconnect macromodeling algorithm. IEEE Trans. Computer-Aided Design, 17(8):645-654, Aug. 1998.

[10] R. Pintelon and J. Schoukens. System Identifi cation: A Frequency Domain Approach. IEEE Press, Inc. New York, 2001.

[11] Y. Rolain, J.Schoukens, and R. Pintelon. Order estimation for linear time-invariant systems using frequency domain identifi cation methods. IEEE Trans. Automatic Control, 1997.

[12] Y. Rolain and R. Pintelon. Generating robust starting values for frequency domain transfer function estimation. IEEE Trans. Automatic Control, 35(5):965-872, May 1999.

[13] Y. Rolain, R. Pintelon, K. Xu, and H. Vold. Best conditioned parametric identifi cation of transfer function models in the frequency domain. IEEE Trans. Automatic Control, 40(11):1954-1960, Nov. 1995.

[14] S. Van Huffel, editor. Recent advances in total least squares techniques and errors-in-variables modeling, chapter Analyses, Development and Applications of TLS-Algorithms in Frequency Domain System Identifi cation, pages 341-358. SIAM, 1997. 